\title{
A Novel Time Reversal-Least Sidelobe Scheme to Minimize ISI and MUI
}

\author{
Do-Hoon Kim, Jungwook Wee, and Kyu-Sung Hwang* \\ Korea Electronics Technology Institute, Seoul, Korea \\ Department of Computer Engineering, Kyungil University, Gyeongbuk, Korea \\ \{speedno, jwwee\}@keti.re.kr, kshwang@kiu.ac.kr
}

\begin{abstract}
In this paper, the scheme that combines a prefilter for sidelobe minimization with the time-reversal prefilter in the ultra wide band wireless communication system is proposed. The proposed scheme can be used in the variable symbol interval situation. When the system has the symbol interval which is larger than one-tap, the proposed scheme exploits the symbol interval to minimize the ISI. In the multi user environment, the proposed scheme is combined with space-time multiplexing scheme to alleviate multi user interference (MUI). Through numerical simulations, we examine the bit error rate (BER) performance of the proposed scheme in single user and two-user environment, and show that the proposed scheme can offer some BER performance gains comparing to the conventional TR scheme.
\end{abstract}

Keywords: UWB communication, Time-reversal, Sidelobe suppression, Additional prefilter.

\section{Introduction}

Recently, in indoor wireless communication environment, ultra wideband (UWB) multimedia wireless communication systems that can transmit high capacity and high speed multimedia data has caught major attentions, because it is able to support high speed transmission of the huge data. However, the UWB operates in a frequency selective fading environment, such that intersymbol interference (ISI) arises and degrades overall performances. In order to overcome the ISI is the time-reversal (TR) scheme in [1] which applies time-reversed complex conjugated channel impulse response (CIR) as a prefilter at transmitter. The TR scheme focuses the signal energy which is scattered by the frequency selective fading to maximize SNR and reduce ISI. In addition, the TR scheme focuses spatial signal energy to the intended receiver; it exploits the channel state information (CSI) to the intended specific position as a prefilter. Therefore, the TR scheme also reduces the multi user interference (MUI). Due to the spatial signal energy focusing property, this scheme has been applied to rich scattering environment, such as a radar system and underwater communication systems $[2,3]$.

\footnotetext{
* Corresponding author.
} 
However, the TR scheme cannot perfectly eliminate ISI due to the residual interference that is remained in the form of sidelobe in the TR equivalent channel impulse response (CIR). In addition, the receiver at the unintended position receives MUI in the form of the cross correlation function between the transmitter to the intended receiver channel and the transmitter to the unintended receiver channel. To mitigate the residual ISI, several schemes have been proposed. In joint TR-ZF scheme [4], zeroforcing (ZF) prefilter was designed in conjunction with the TR prefilter to eliminate the residual ISI and maximize SNR. The schemes in $[5,6]$ exploit the symmetric property of the equivalent CIR's power to reduce the multi-user interference (MUI). In these schemes, each transmitter sends the signal, which is shifted to each other to avoid the peak-power interference from another transmitter.

In this paper, we propose a novel structure of an additional prefilter at the transmitter to suppress the residual ISI and reduce the complexity of the receiver. We consider a variable rate system that the symbol interval can be adjustable. The proposed scheme exploits the symbol interval to suppress ISI in conjunction with semidefinite relaxation (SDR) [7] technique to solve optimization problem of minimizing ISI with some constraints. The following notations are used for description throughput this paper. The operators $*,(\cdot)^{H},(\cdot)^{*}$, and $\|\cdot\|$ indicate convolution, Hermitian transpose, complex conjugation, and norm operators respectively.

\section{System Models}

\subsection{Indoor Wireless Channel Model}

In this paper, we consider an exponential decay model in [8] for an indoor wireless channel model in order to simplify the representation and the performance analysis which is useful to express a typical frequency selective fading channel. The power delay profile (PDP) of an exponential decay model indicates the mean power at the $l^{\text {th }}$ tap and can be expressed as

$$
\sigma_{1}^{2}=\exp \left(\frac{(l-1) \Delta r}{\bar{\sigma}_{\tau}}\right)
$$

where $\Delta \tau$ is an interval between adjacent taps, and $\bar{\sigma}_{\tau}$ represents the mean RMS delay spread. The coefficient at the $l^{\text {th }}$ tap of the channel impulse response is an identically and independently distributed circularly complex Gaussian random variable with zero mean and $\sigma_{l}^{2}$ variance.

\subsection{Single User TR-LS System}

Firstly, we consider a single user system that employs a TR prefilter at the transmitter side. The main advantage of this scheme is that it maximizes the transmit signal to noise ratio (SNR) since the CSI of the receiver is available at the transmitter while also greatly reducing the receiver's complexity by only employing a single-tap 
equalizer. However, the drawback of the TR scheme is that it has a residual ISI. The TR scheme makes an equivalent channel impulse response which is the original CIR's autocorrelation function that has maximum peak point and symmetrical sidelobes. The TR scheme maximizes the SNR but does not remove ISI completely. Thus, the performance of the system will be degraded by the residual ISI. To suppress the residual ISI, this paper proposes a scheme that employs an additional prefilter at the transmitter. The transmitter has two filters: one is a conventional TR prefilter while the other is a least sidelobe (LS) filter that minimizes the residual ISI from the TR filter. From the assumption of TR-LS system, the received signal can be written as

$$
y(t)=f^{T R}(t) * g^{L S}(t) * s(t)+n(t)
$$

where $s(t)$ is a message signal at time $t, g^{L S}(t)$ is the LS filter with length $2 L-1$ and $f$ ${ }^{T R}(t)$ is the TR equivalent channel that can be expressed as

$$
f^{T R}(t)=h(t) * g^{T R}(t)
$$

The TR prefilter $\mathrm{g}^{T R}$ can be represented as

$$
g^{T R}(t)=\frac{h^{*}(-t)}{\|h(t)\|}
$$

where $h^{*}(-t)$ is time-reversed and conjugated form of the $h(t)$. In (4), the nominator means a conjugation of the time-reversed channel impulse response. Variable $n(t)$ is an additive zero-mean complex Gaussian noise. We can rewrite (3) in its discrete representation

$$
f^{T R}[k]=h[k]^{*} g^{T R}[k]
$$

where $k$ is a discrete sampling instant. The entire transmit prefilter is a combination of the TR filter and the LS filter which can be represented as

$$
g^{T R-L S}[k]=g^{L S}[k]^{*} g^{T R}[k]
$$

where $g^{L S}[k]$ is a prefilter proposed in this paper. The prefilter $g^{L S}[k]$ performs peak power reduction and sidelobe expansion to minimize residual ISI. We consider the LS filter with length of $2 L-1$, similar to the conventional TR equivalent channel impulse response's length. The equivalent entire system channel impulse response $f^{T R-L S}[k]$ can be represented as

$$
f^{T R-L S}[k]=h[k] * g^{T R-L S}[k] .
$$

\subsection{Two User TR-LS System}

For the two user TR-LS system, we consider a two transmitter-receiver pairs in the indoor wireless communication environment which can cause interference to each other. We assume the CSI of the intended receiver are available at each transmitter. However, the CSI of unintended receiver is unavailable. Since the transmitters have 
only the CSI of the intended user, each transmitter generate the prefilters using only the intended receiver's channels, respectively. To avoid the MUI, we introduce a scheme which combines the shifted transmission technique in [5] and the adjustment of the mainlobe's width.

Based on the proposed two user TR-LS system, the received signal at receiver1 can be written as

$$
y_{1}[k]=f_{11}^{T R}[k]^{*} g_{1}^{L S}[k]^{*} s_{1}[k]+f_{21}^{T R}[k]^{*} g_{2}^{L S}[k] * s_{2}[k]+n_{1}[k]
$$

where

$$
f_{11}^{T R}[k]=h_{11}[k]^{*} g_{1}^{T R}[k]
$$

and

$$
f_{21}^{T R}[k]=h_{21}[k]^{*} g_{2}^{T R}[k]
$$

The equivalent channel impulse response (10) can be called an interference chanel. Variable $g_{1}^{L S}[k]$ and $g_{2}^{L S}[k]$ are the least sidelobe prefilter at the transmitter 1 and 2 , respectively, while $s_{1}$ and $s_{2}$ are the transmitted symbol at the transmitter 1 and 2 , respectively. Similarly, the received signal at receiver 2 can be described as

$$
y_{2}[k]=f_{22}^{T R}[k]^{*} g_{2}^{L S}[k] * s_{1}[k]+f_{21}^{T R}[k]^{*} g_{1}^{L S}[k]^{*} s_{1}[k]+n_{2}[k]
$$

where

$$
f_{22}^{T R}[k]=h_{22}[k] * g_{2}^{T R}[k]
$$

and

$$
f_{12}^{T R}[k]=h_{12}[k]^{*} g_{1}^{T R}[k]
$$

In (8) and (11), the first term is the intended signal, the second term is MUI, and the third term is an additive zero-mean complex gaussian noise.

\section{TR-LS Scheme for ISI Suppression}

This section describes out proposed scheme that exploits an additional prefilter at the transmitter to minimize the residual ISI in the TR wireless communication system. To achieve this, following subsections explain how to design the additional prefilter that minimize the sidelobe of TR equivalent impulse response.

\subsection{Design of the Least Sidelobe Prefilter}

Optimization Problem. Firstly, we examine how to design the least sidelobe prefilter $g^{L S}(t)$ that is designed by an optimization theory. The prefilter $g^{L S}(t)$ can be described 
as the matrix form, $\mathrm{g}^{L S}$. The objective of the prefilter is the minimization of residual ISI. Therefore, the optimization problem can be represented as

$$
\begin{gathered}
\underset{\mathbf{g}^{L S}}{\operatorname{minimize}}\left\|\tilde{\mathbf{F}}^{T R} \mathbf{g}^{L S}\right\|^{2} \\
\text { subject to } \\
\left\|\mathbf{f}^{\operatorname{Rev}} \mathbf{g}^{L S}\right\|^{2} \geq c ; 0 \leq c \leq 1 \\
\left\|\mathbf{G}^{T R} \mathbf{g}^{L S}\right\|^{2}=1
\end{gathered}
$$

where $\tilde{\mathbf{F}}^{T R}$ is a matrix that represents ISI components to be minimize, and $c$ is a peak power constraint that has a relative value to the peak power of original TR scheme. $\mathbf{f}^{T R}$ is a TR equivalent channel impulse response and $\mathbf{f}^{\mathrm{Rev}}$ is a reversed vector of $\mathbf{f}^{T R}$. The matrix $\tilde{\mathbf{F}}^{T R}$ is a modification of the original matrix $\mathbf{F}^{T R}$. The matrix $\mathbf{F}^{T R}$ represents a TR equivalent channel.

$$
\mathbf{F}^{T R}=\left[\begin{array}{cccc}
f^{T R}[1] & 0 & \cdots & 0 \\
f^{T R}[2] & f^{T R}[1] & & \vdots \\
\vdots & \vdots & \ddots & 0 \\
f^{T R}[2 L-1] & f^{T R}[2 L-2] & & f^{T R}[1] \\
0 & f^{T R}[2 L-1] & & f^{T R}[2] \\
\vdots & 0 & \ddots & \vdots \\
0 & \vdots & & f^{T R}[2 L-1]
\end{array}\right],
$$

and $\mathbf{g}^{L S}$ is a solution of this optimization problem. This solution is a vector form of the least sidelobe prefilter.

Focusing the Residual ISI within Symbol Interval. In (14), the solution minimizes ISI term. The ISI term is represented by $\tilde{\mathbf{F}}^{T R}$. This term has to be minimized by $\mathbf{g}^{L S}$. $\tilde{\mathbf{F}}^{T R}$ is a modified matrix of the equivalent TR channel matrix $\mathbf{F}^{T R}$.

In (14), central rows are nulled with zeros. Rows of $\tilde{\mathbf{F}}^{T R}$ are multiplied with $\mathbf{g}^{L S}$. Then, we can get a vector that represents ISI. The optimization solution $\mathbf{g}^{L S}$ minimizes ISI power. Thus, the multiplication of $\tilde{\mathbf{F}}^{T R}$ and $\mathbf{g}^{L S}$ has to be minimized by the solution. This is expressed by (14). When we compose $\tilde{\mathbf{F}}^{T R}$, we have to consider the focusing of ISI within the symbol interval. Thus, the solution minimizes only the symbol interval tap and outside of the symbol interval. To represent the outside of the symbol interval, we need nulling the central rows of $\tilde{\mathbf{F}}^{T R}$. Then central taps of $\tilde{\mathbf{F}}^{T R} \mathbf{g}$ are omitted in the minimization target of the solution. Thus, in an equivalent channel impulse response, the taps inside of the symbol interval are not minimized. But they are not our concerns, because the taps do not interfere sampling time instants. The ignoring taps inside of symbol interval leads increase of the degree of freedom. Thus, the solution can exploit the increase of the degree of freedom to the suppressing ISI power. If the sytem gets more taps inside of symbol interval, more ISI power can be suppressed. 


\subsection{Conversion to Solvable Form}

This subsection deals with the process to convert the optimization problem to solvable form. The optimization problem which discussed in previous section can be converted to a quadratic form. (14) can be converted as

$$
\begin{aligned}
& \underset{\mathbf{g}^{L S}}{\operatorname{minimize}} \quad\left(\mathbf{g}^{L S}\right)^{H} \mathbf{A} g^{L S} \\
& \text { subject to }\left(\mathbf{g}^{L S}\right)^{H} \mathbf{B} g^{L S} \leq c ; 0 \leq c \leq 1 \\
& \left(\mathbf{g}^{L S}\right)^{H} \mathbf{C} g^{L S}=1
\end{aligned}
$$

where $\quad \mathbf{A}=\left(\tilde{\mathbf{F}}^{T R}\right)^{H} \tilde{\mathbf{F}}^{T R}, \mathbf{B}=\left(\mathbf{f}^{\mathrm{Re} v}\right)^{H} \mathbf{f}^{\mathrm{Rev}}$, and $\mathbf{C}=\left(\mathbf{G}^{T R}\right)^{H} \mathbf{G}^{T R}$.

Now, the optimization problem is a quadratically constrained quadratic programming (QCQP). However, despite the conversion, the optimization problem is nonconvex, because the second constraint in (16) is a quadratic form equality constraint that is a non-convex. We can use the semi-definite programming relaxation (SDR) [7] to convexity of the optimization problem. First, the QCQP has to convert to semidefinite programming (SDP). Thus, (16) can be reformulated as

$$
\begin{array}{cc}
\underset{\mathbf{G}^{L S}}{\operatorname{minimize}} & \operatorname{trace}\left(\mathbf{A G}^{L S}\right) \\
& \operatorname{trace}\left(\mathbf{B G}^{L S}\right) \leq c ; 0 \leq c \leq 1 \\
\text { subject to } & \operatorname{trace}\left(\mathbf{C G}^{L S}\right)=1 \\
& \operatorname{rank}\left(\mathbf{G}^{L S}\right)=1 \\
& \mathbf{G}^{L S} \succeq 0
\end{array}
$$

(17) is a non-convex SDP, because the constraint $\operatorname{rank}\left(\mathbf{G}^{L S}\right)=1$ is not a convex. By the relaxation of this constraint, we can change the non-convex SDP to the convex SDP. The changed optimization problem can be described as

$$
\begin{array}{cc}
\underset{\mathbf{G}^{L S}}{\operatorname{minimize}} & \operatorname{trace}\left(\mathbf{A G}^{L S}\right) \\
& \operatorname{trace}\left(\mathbf{B G}^{L S}\right) \leq c ; 0 \leq c \leq 1 \\
\text { subject to } & \operatorname{trace}\left(\mathbf{C G} \mathbf{G}^{L S}\right)=1 \\
& \mathbf{G}^{L S} \succeq 0
\end{array}
$$

This optimization problem can be solved by the convex SDP that can derive the solution as matrix form, $\mathbf{G}^{L S}$. However we want a solution of the vector form, $\mathbf{G}^{L S}$. The vector form solution can be extracted from $\mathbf{G}^{L S}$ by a rank-1 approximation. To extract $\mathbf{g}^{L S}$, let

$$
\mathbf{G}^{L S}=\sum_{i=1}^{r} \lambda_{i} \mathbf{q}_{i} \mathbf{q}_{i}{ }^{H},
$$

where $r$ is a rank of $\mathbf{G}^{L S}$, and the eigenvalues of $\mathbf{G}^{L S}$ are $\lambda_{1} \geq \lambda_{2} \geq \ldots \geq \lambda_{\mathrm{r}}>0$, and $\mathbf{q}_{1}, \ldots, \mathbf{q}_{\mathrm{r}}$ are the eigenvectors of $\mathbf{G}^{L S}$. We choose maximum eigenvalue and its eigenvector, $\mathbf{g}^{L S}=\sqrt{\lambda_{1}} \mathbf{q}_{1}$. This is a solution of the optimization problem. 


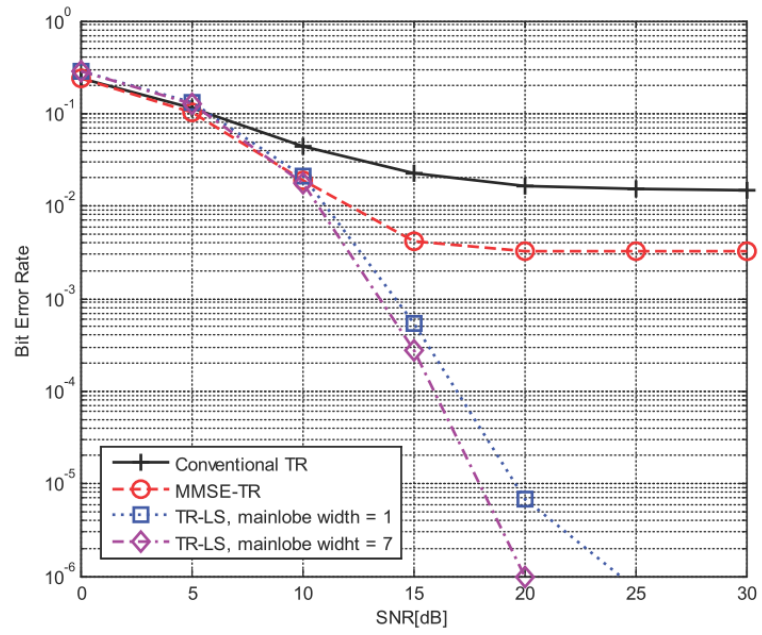

Fig. 1. BER performance comparison between the variable mainlobe width $\mathrm{W}_{m}=1,7$, and conventional schemes

\subsection{Single User System}

In single user system which doesn't have MUI, reduction of the sidelobe can be achieved by handling the mainlobe width. To minimize ISI, we exploit the symbol interval. In previous section, we examined the wider mainlobe leads to lower sidelobe. However, if the expanded mainlobe interferes the adjacent sampling instance, ISI is occurred severely. Thus, the mainlobe width will set to a little bit shorter than adjacent sampling instance to avoid ISI from the mainlobe, therefore the mainlobe doesn't interfere to the adjacent sampling instance. The mainlobe width can be set $W_{m}$ $=2 T_{s}-1$ where $W_{m}$ is a mainlobe width and $T_{s}$ is a symbol interval.

\subsection{Two User System}

In Two user system, we have to pay attention to MUI which cannot handle by the TRLS prefilter due to the prefilter does not have the CSI to unintended receiver. Thus, in this paper, the proposed scheme is combined with the space time multiplexing scheme that explained in the previous chapter. To overcome MUI, the optimization problem of the TR-LS prefilter has to set the high $c$ constraint, because the transmitter cannot mitigate MUI. Thus, the intended channel has to secure a measure of the peak power to overcome MUI. Also, the shifted transmission scheme can alleviate MUI [5].

\section{Simulation Results}

In this section, we provide the BER performance of the proposed TR-LS with QPSK and compare that with the conventional schemes. The simulation parameters used 


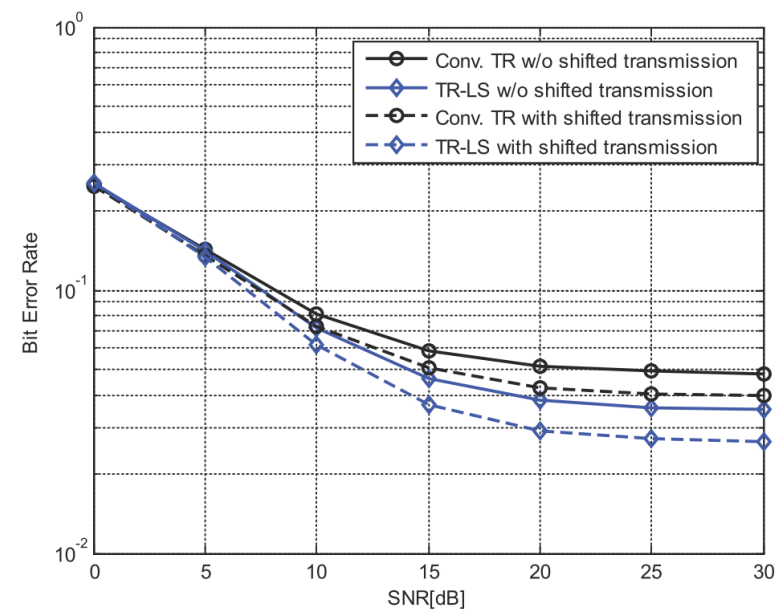

Fig. 2. BER performance comparison between the conventional TR scheme and the proposed TR-LS scheme with $W_{m}=2$

in this paper is given by the channel impulse response $L=38, \Delta \tau=0.811 \mathrm{~ns}$, and 100 symbols of block length. In two user environment, the space-time multiplexing scheme [5] is applied and the minimum symbol interval of 2 is used.

Figure 1 shows the comparison of BER performance in a single user environment between the proposed scheme, the conventional TR, and MMSE-TR in [9] with the fixed symbol interval, and variable mainlobe width $\mathrm{W}_{m}$. As the mainlobe is broadening, the BER performance is improved. As shown in Figure 1, the proposed scheme has better BER performance than conventional schemes. Figure 2 gives the BER performance of the two user system. In this simulation, we set $c^{\prime}=0.9$. The result shows that TR-LS scheme has better BER performance than conventional TR scheme and shifted TR scheme both. Since TR-LS scheme is combined with shifted transmission scheme, BER performance is slightly improved. As shown in Figure 2, he proposed scheme reduces bit error by 26 percent as compared with conventional TR scheme.

\section{Conclusion}

In this paper, we proposed the TR-LS scheme to minimize ISI and MUI in UWB communication system. In the frequency selective fading channel environment, the proposed scheme exploits the symbol interval to minimize the residual ISI in the TR communication system. Since the system broadens the symbol interval, the TR-LS scheme can reduce more ISI. In addition, the proposed scheme expands the mainlobe width to reduce ISI. The expanded mainlobe width makes lower sidelobe power. Thus, the proposed scheme shows an improved signal to-ISI ratio and BER performance. From some selected simulation results, the proposed scheme shows outperform the conventional schemes. 
Acknowledgements. This work was supported by the IT R\&D program of MKE/KEIT.

\section{References}

1. Fink, M.: Time-reversed Acoustic. Scientific American, 67-73 (1999)

2. Jin, Y., Moura, J.M.F., Jiang, Y., Stancil, D.D., Cepni, A.G.: Time Re-versal Detection in Clutter: Additional Experimental Results. IEEE Trans. Aerospace and Electronic Systems 47, 140-154 (2011)

3. Edelmann, G.F., Song, H.C., Kim, S., Hodgkiss, W.S., Kuperman, W.A., Akal, T.: Underwater Acoustic Communications using Time Reversal. IEEE Journal of Oceanic Engineering 30, 852-864 (2005)

4. Kyritsi, P., Stoica, P., Papanicolaou, G., Eggers, P., Oprea, A.: Time Re-versal and ZeroForcing Equalization for Fixed Wireless Access Channels. In: Asilomar Conference on Signals, Systems and Computers (2005)

5. Nguyen, H.T., Kovacs, I.Z., Eggers, P.C.F.: Time Reversal Technique for Multi-user Wireless Communication with Single Tap Receiver. Mobile and Wireless Communications Summit 16, 1-5 (2007)

6. Sigit, A.P., Choi, S.K., Lee, C.Y.: Shifted Time Reversal Technique for Two-user Wireless Communication Using Variable Rate Back-off. IEEK Journal 5, 33-39 (2011)

7. Luo, Z.-Q., Ma, W.-K., So, A.M.-C., Ye, Y., Zhang, S.: Semidefinite Re-laxation of Quadratic Optimization Problems. EEE Signal Processing Magazine 27, 20-34 (2010)

8. Vaughan, R., Andersen, J.B.: Channels Propagation and Antennas for Mobile Communications. IEE Electromagnetic Waves Series, vol. 50 (2003)

9. Strohmer, T., Emami, M., Hansen, J., Papanicolaou, G., Paulraj, A.J.: Application of TimeReversal with MMSE Equalizer to UWB Communications. In: Global Telecommunications Conference, GLOBECOM 2004, vol. 5, pp. 3123-3127. IEEE (2004) 